\title{
MODELOS GEOMÉTRICOS EN EL ESTUDIO DE NANOTUBOS DE CARBONO
}

\author{
Guillermo A. Guirales ${ }^{1}$ \\ RICARdo PÉREZ ${ }^{2}$ \\ Boris A. RODRÍGUEZ ${ }^{3}$
}

\section{Resumen}

Los nanotubos de carbono se han convertido en las últimas dos décadas en elementos de gran interés tanto para investigaciones teóricas como para desarrollos tecnológicos. Desde el punto de vista teórico, para su estudio es necesario un modelo que describa lo mejor posible sus propiedades físicas. Por lo tanto es importante realizar una aproximación a la geometría que posibilite su descripción física. En el presente artículo además de una revisión de los modelos geométricos del nanotubo, se realizará una descripción de algunos de los desarrollos tecnológicos más interesantes. En los modelos teóricos de descripción de la geometría del nanotubo, el primer método es el llamado modelo de malla enrollada. Este consiste en tratar el nanotubo como una porción de malla de grafeno a la cual se le da unas condiciones de periodicidad, para que al enrollarla reproduzca las posiciones de los átomos de carbono. El segundo método, consiste en plantear los átomos en el nanotubo como lugares en una estructura cilíndrica. En este modelo los vectores que se usan para la descripción se sacan de la geometría

1 Grupo de Física Atómica y Molecular, Universidad de Antioquia. Docente ocasional de ciencias básicas, ITM, Medellín-Colombia, guillermoguirales@itm.edu.co

2 Department of Engineering Physics, McMaster University, Ontario-Canadá, perezcr@mcmaster.ca

3 Grupo de Física Atómica y Molecular, Universidad de Antioquia, Medellín-Colombia, banghelo@fisica.udea.edu.co. 
particular de cada nanotubo y escribiéndolos en coordenadas cilíndricas. Finalmente, el tercer método, se fundamenta en la descripción del nanotubo a partir de todas sus posibles simetrías; tratando al nanotubo como una estructura que posee una simetría particular a lo largo de una dirección.

\section{Palabras clave}

Nanotubo de carbono, malla enrollada, coordenadas cilíndricas, teoría de grupos, nanotecnología.

\section{Abstract}

Carbon nanotubes have become in the last two decades in greatest elements both in theoretical investigation as technological developments. From theoretical point of view, a model is necessary that describe on the best possible its physical properties, thus an approximation to the geometry is important for a physical description. In this paper in addition to a geometric model revision to the nanotube, we are going to do a description to the most interesting technological developments. In the theoretical models of nanotube geometric description, the first is the called zone folding method. This method consist in deal the nanotube like a mesh piece of graphen to which is given a periodicity conditions, so when it is folded it reproduce all the carbon atoms positions. The second method, consist in propose the atoms in the nanotube like places in a cylindrical structure. In this model the vectors used to the description are obtained from the particular geometry in each nanotube and write them in cylindrical coordinates. Finally, the third method is based in the nanotube description from all its possible symmetries, dealing the nanotube as a structure that has a particular symmetry along one direction.

\section{Keywords}

Carbon nanotube, zone folding, cylindrical coordinates, group theory, nanotechnology. 


\section{INTRODUCCIÓN}

El descubrimiento experimental de los nanotubos de carbono fue hecho en 1991, cuando Sumio Iijima (Iijima, 1991) del laboratorio de Investigación Fundamental de NEC en Tsukuba, observaba unas extrañas fibras nanoscópicas depositadas sobre una mota de hollín. Constituidas por carbono, y de forma tan regular y simétrica como los cristales; las nano-estructuras de carbono se han convertido en un elemento interesante tanto para las ciencias como para la ingeniería (Stix, 2005; Rivas et al., 2007). Muchas son las propiedades extraordinarias de los nanotubos por ejemplo su gran elasticidad, resistencia a la tracción y estabilidad térmica, lo que los convierte en objetos con interesantes propiedades mecánicas y electrónicas para el diseño de nuevos materiales y dispositivos. Los primeros nanotubos de carbono que observó Iijima se denominaron Nanotubos de Paredes Múltiples; cada uno contenía cierto número de cilindros huecos de átomos de carbono concéntricos como el tronco de un árbol.

Dos años después, Iijima y Donald Bethune, de IBM (Bethune et $a l ., 1993)$, crearon cada uno por su lado, nanotubos de Pared Única, formados por una sola capa de átomos de carbono. Ambos tipos de tubos, fabricados de modo parecido, gozan de varias propiedades similares. El modelo de pared única, de un nanómetro aproximado de diámetro, puede medir miles de nanómetros de longitud. En los nanotubos, los átomos se disponen en anillos hexagonales, la misma estructura que caracteriza al grafito; de hecho un nanotubo parece una lámina de grafito enrollada en un cilindro sin costuras. Una de las particularidades más importantes del grafito y que lo ubican como material de gran interés para las ingenierías es que éste pertenece al grupo restringido de los semimetales. Combinando las propiedades semimetálicas del grafito con las reglas de selección de los niveles de energía en el nanotubo, se logran obtener conductores exóticos a partir de los nanotubos de carbono (Saito, 1998).

El presente trabajo considera una revisión de los modelos teóricos más relevantes en el estudio de los nanotubos de carbono, además de realizar un análisis del impacto tecnológico que significa el desarrollo de estos dispositivos. 


\section{Modelos teóricos}

Los átomos de carbono en presencia de otros átomos tienden a realizar enlaces covalentes, a esta acción se le llama hibridación del átomo (Muntaner, 1972; Saito, 1998). El carbono tiene seis electrones ocupando los orbitales $1 s^{2}, 2 s^{2}$ y $2 p^{2}$. El orbital $1 s^{2}$ es extremadamente ligado al átomo, mientras que los menos ligados son los $2 s$ y $2 p$, los cuales son los orbitales disponibles para realizar enlaces covalentes.

El orbital $2 p$ se divide en $2 p_{x}, 2 p_{y}$ y $2 p_{z}$. En nuestro caso nos interesa la hibridación $s p^{2}$, la cual corresponde a la forma geométrica como se conectan los átomos de carbono en un nanotubo. En la hibridación $s p^{2}$ los orbitales se entremezclan de tal forma que los máximos de probabilidad de la función de onda de los electrones se localizan formando un ángulo de $120^{\circ}$, si suponemos que el estado de los electrones que representa cada orbital se escribiera como $|2 s\rangle$, para los electrones en el orbital y $2 s$ y $\left|2 p_{x}\right\rangle,\left|2 p_{y}\right\rangle$ para los electrones en el orbital $2 p$; entonces cada orbital hibridizado está descrito desde (1) hasta (3).

$$
\begin{gathered}
\left|s p_{a}^{2}\right\rangle=\frac{1}{\sqrt{3}}|2 s\rangle-\sqrt{\frac{2}{3}}\left|2 p_{y}\right\rangle \\
\left|s p_{b}^{2}\right\rangle=\frac{1}{\sqrt{3}}|2 s\rangle+\sqrt{\frac{2}{3}}\left(\frac{\sqrt{3}}{2}\left|2 p_{x}\right\rangle+\frac{1}{2}\left|2 p_{y}\right\rangle\right) \\
\left|s p_{c}^{2}\right\rangle=\frac{1}{\sqrt{3}}|2 s\rangle+\sqrt{\frac{2}{3}}\left(-\frac{\sqrt{3}}{2}\left|2 p_{x}\right\rangle+\frac{1}{2}\left|2 p_{y}\right\rangle\right)
\end{gathered}
$$

En esta configuración siempre queda un electrón, llamado electrón que se mantiene radial al nanotubo, el cual es el responsable de los fenómenos de transporte eléctrico, de interacción con otros nanotubos y efectos ópticos de interacción con la luz; como sólo hay un electrón por átomo de carbono entonces se simplifica el lenguaje hablando sólo del átomo de carbono. Esto nos proporciona una idea de cómo se conectan los átomos de carbono, las características que 
se tiene cuando se realizan estas conexiones nos la da la teoría de grupos. Los átomos de carbono al conectarse en la hibridación $2 p^{2}$, posibilitan el hecho de que se formen ciertas estructuras periódicas y que además poseen una connotación geométrica bastante fuerte, es decir, son altamente simétricos.

Una de las primeras observaciones de estas simetrías son las que dan el nombre a los nanotubos; al hacer un corte transversal al eje del nanotubo se puede ver que los átomos se conectan formando un zigzag, por lo tanto a estos se les llama nanotubos zigzag. En otro tipo al hacer este corte se observa una conexión semejante al brazo de un sillón, por eso se les llama nanotubos brazo de sillón. Y un tercer tipo no se reconoce algún patrón en particular, a estos se les llama nanotubos quirales.

\subsection{Malla enrollada}

Para iniciar un estudio geométrico de la estructura se definen dos vectores $\vec{a}_{1}$ y $\vec{a}_{2}$ sobre la superficie de grafeno como lo realiza (Barros et al., 2006). Estos son vectores que unen los centros de los hexágonos vecinos (ver Fig. 1), con la particularidad de que $a_{1}=a_{2}$ $=a_{c}$, la magnitud de los vectores y con $a_{c}$ la longitud característica de los hexágonos de carbono; además de la geometría se ve que $\vec{a}_{1} \cdot \vec{a}_{2}=a_{C}^{2} / 2$.

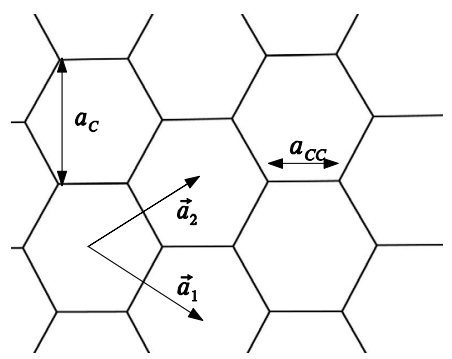

a)

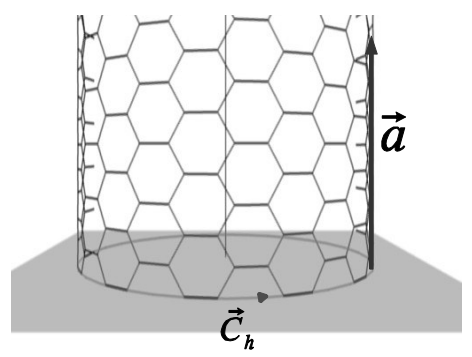

b)

Fig. 1. A) Representación de Los Vectores primitivos del nanotubo, JUNTO CON LA LONGITUD CARACTERÍSTICA ENTRE ÁtOMOS DE CARBONO $a_{C C}=1,42 \AA$ Y LA CONSTANTE DE RED $a_{C}=2,46 \AA$. B) REPRESENTACIÓN DE LOS VECTORES QUIRAL $\overrightarrow{c_{h}}$ Y DE TRASLACIÓN $\vec{a}$. 
La descripción del nanotubo se hace a partir del vector $\overrightarrow{C_{h}}$, llamado vector quiral y del vector $\vec{a}$, llamado vector de traslación. La magnitud del vector $\overrightarrow{C_{h}}$ mide igual que el perímetro del nanotubo y se escribe como combinación lineal de los vectores primitivos:

$$
\overrightarrow{C_{h}}=n_{1} \vec{a}_{1}+n_{2} \vec{a}_{2}
$$

Igualmente el vector de translación se define como

$$
\vec{a}=t_{1} \vec{a}_{1}+t_{2} \vec{a}_{2}
$$

que bajo la condición de perpendicularidad $\vec{C}_{h} \cdot \vec{a}=0$ se obtiene que $t_{1}=-\left(2 n_{2}+n_{1}\right) / n R$ y $t_{2}=\left(2 n_{1}+n_{2}\right) / n R$, con el máximo común divisor (MCD) entre $n_{1}$ y $n_{2}$, es decir $n=M C D\left[n_{1}, n_{2}\right]$. $R$ un número entero tal que $R=1$, si el número $\left(n_{1}-n_{2}\right)$ no es múltiplo entero de $3 n$; o $\mathrm{R}=3$, si el número $\left(n_{1}-n_{2}\right)$ es múltiplo entero de $3 n$. Los dos vectores $\overrightarrow{C_{h}}$ y $\vec{a}$ definen el nanotubo unitario, entonces el número de pares de átomos de carbono en el nanotubo unitario es como

$$
Q=\frac{\left|\vec{C}_{h} \times \vec{a}\right|}{\left|\vec{a}_{1} \times \vec{a}_{2}\right|}=\frac{2\left(n_{1}^{2}+n_{2}^{2}+n_{1} n_{2}\right)}{n R}
$$

El radio del nanotubo y la magnitud del vector de traslación están dados en (7) y (8) respectivamente.

$$
\begin{gathered}
r_{t}=\frac{a_{c}}{2 \pi} \sqrt{\frac{n R Q}{2}} \\
a=a_{c} \sqrt{\frac{3 Q}{2 n R}}
\end{gathered}
$$

Este método trata entonces al nanotubo como una malla de grafeno limitado por los vectores $\overrightarrow{C_{h}} \mathrm{y} \vec{a}$, la cual luego de enrollarse sobre el vector $\overrightarrow{C_{h}}$ reproduce el nanotubo unitario. 


\subsection{Coordenadas cilíndricas}

Otro modelo se basa en la construcción geométrica del nanotubo de carbono en coordenadas cilíndricas (Zang, 2005). En este modelo se define los vectores de la red recíproca, con el fin de determinar la estructura de bandas de energía más fácilmente y así poder construir el Hamiltoniano.

Para el nanotubo brazo de sillón se tienen los vectores

$$
\begin{gathered}
\vec{b}_{\varphi}=\frac{2 \pi}{\Omega} \vec{a}_{z} \times \hat{e}_{r}=\frac{2 \pi}{\Omega} a \hat{e}_{\varphi} \\
\vec{b}_{p}=\frac{2 \pi}{\Omega} \vec{a}_{p} \times \hat{e}_{r}=-\frac{2 \pi}{\Omega}\left(\frac{3 r_{0}}{2} \hat{e}_{z}-\frac{a}{2} \hat{e}_{\varphi}\right) \\
\vec{k}=k_{z} \hat{e}_{z}+k_{\varphi} \hat{e}_{\varphi}
\end{gathered}
$$

En donde cada uno de los vectores está en coordenadas cilíndricas y para este nanotubo particular $\Omega=\left|\hat{e} \cdot \vec{a}_{p} \times \vec{a}_{z}\right|$ es el volumen de la celda unitaria, $r_{o}$ es el radio del nanotubo brazo de sillón y las componentes del vector de onda son

$$
\begin{gathered}
k_{\varphi}=\frac{m}{r_{0}}, m=0, \pm 1, \pm 2, \ldots, \pm N_{a r} \\
-\frac{4 \pi}{3 a} \leq k_{z} \leq \frac{4 \pi}{3 a}
\end{gathered}
$$

En (12), $N_{a r}$ representa el número de átomos en el perímetro del nanotubo, por último $r_{o}$ es:

$$
r_{0}=\frac{\sqrt{5+4 \cos \left(2 \pi / N_{a r}\right)}}{2 \sqrt{3} \operatorname{sen}\left(2 \pi / N_{a r}\right)} a
$$

De igual forma para un nanotubo zigzag se tienen los vectores 


$$
\begin{gathered}
\vec{b}_{z}=\frac{2 \pi}{\Omega^{\prime}} \hat{e}_{r} \times \vec{a}_{\varphi}=\frac{2 \pi}{\Omega^{\prime}} a \hat{e}_{z} \\
\vec{b}_{q}=\frac{2 \pi}{\Omega^{\prime}} \hat{e}_{r} \times \vec{a}_{q}=\frac{2 \pi}{\Omega^{\prime}}\left(\frac{3 r_{0}^{\prime}}{2} \hat{e}_{z}-\frac{a}{2} \hat{e}_{\varphi}\right) \\
\vec{k}=k_{z} \hat{e}_{z}+k_{\varphi} \hat{e}_{\varphi}
\end{gathered}
$$

En donde cada uno de los vectores está en coordenadas cilíndricas y para este nanotubo particular $\Omega^{\prime}=\left|\hat{e}_{r} \cdot\left(\vec{a}_{\varphi} \times \vec{a}_{q}\right)\right|$ es el volumen de la celda unitaria. El radio del nanotubo zigzag $r_{o}^{\prime}$ y las componentes del vector de onda están dados por

$$
\begin{gathered}
k_{\varphi}=\frac{n}{r_{0}^{\prime}}, n=0, \pm 1, \pm 2, \ldots, \pm N_{z i} / 2 \\
-\frac{2 \pi}{a \sqrt{3}} \leq k_{z} \leq \frac{2 \pi}{a \sqrt{3}} \\
r_{0}^{\prime}=\frac{a}{\operatorname{sen}\left(2 \pi / N_{z i}\right)}
\end{gathered}
$$

Donde en las expresiones anteriores $N_{z i}$ representa el número de átomos en el perímetro del nanotubo. Una representación de estos vectores está en la Fig. 1.

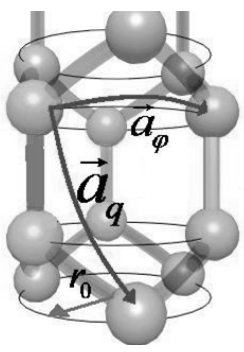

a)

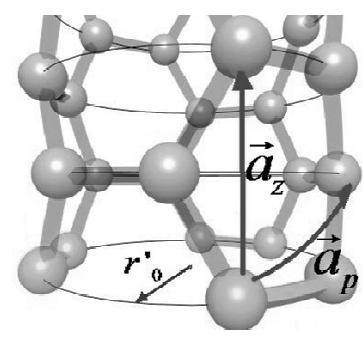

b)

FIG. 1. REPRESENTACIÓN DE LOS VECTORES DEFINIDOS EN COORDENADAS CILÍNDRICAS PARA A) UN NANOTUBO ZIGZAG Y B) UN NANOTUBO BRAZO DE SILLÓN 


\subsection{Grupos de línea}

Un grupo de línea debe pensarse como un conjunto cuyos elementos son una serie de operaciones de simetría a lo largo de un eje principal. La diferencia del grupo de línea con los grupos puntuales, rotaciones, reflexiones, inversiones (Tinkham, 1964; Naussbaum, 1975) es que el grupo de línea es un grupo más grande que contiene los grupos puntuales además de una simetría de traslación. El grupo de línea se ha usado principalmente para el estudio de moléculas estéreo regulares (Božović \& Vujičić, 1978; 1981).

Por más largo que sea un nanotubo siempre se puede reducir a un conjunto más pequeño de átomos que al moverlos a lo largo del eje del nanotubo, se reconstruye el nanotubo completamente. Se concluye que los elementos del grupo de línea encargados de reconstruir el nanotubo a partir de un átomo son

$$
\left\{l_{t s u}\right\}=\left\{\left(C_{Q}^{r} \mid \frac{n \vec{a}}{Q}\right)^{t} C_{n}^{s} U^{u}\right\}
$$

El término $\left(C_{Q}^{r} \mid \frac{n \vec{a}}{Q}\right)^{t}$ representa las operaciones de torsión, $C_{n}^{S}$ representa las operaciones de rotación pura y $U^{u}$ las operaciones de reflexión como se ilustra en la Fig. 2. $n$ es el máximo común divisor entre $n_{1}$ y $n_{2}, Q$ está dado por (6) y $r$ está dado por la función

$$
r=\frac{Q}{n} F r\left[\frac { 2 } { Q R } \left(3-\frac{2\left(n_{1}-n_{2}\right)}{n_{1}}+\frac{n}{n_{1}}\left(\frac{n_{1}-n_{2}}{n}\right)^{\varphi\left(\frac{n_{1}}{n}\right)-1}\right.\right.
$$

En donde $\operatorname{Fr}[x]$ es la parte fraccionaria de $x$ y $\varphi(m)$ es la función de Euler que da el número de enteros menores o iguales que $m$ y coprimos con $m$. Se concluye, usando los elementos definidos en (21), que la posición de un átomo puede proveer el resto de posiciones de los átomos que forman el nanotubo independiente de la familia a la que pertenezca mediante la función 


$$
r_{t s u}=\left(\rho,(-1)^{u} \phi_{0}+2 \pi\left(\frac{r t}{Q}+\frac{s}{n}\right),(-1)^{u} z_{0}+\frac{t n a}{Q}\right)
$$

la cual está en coordenadas cilíndricas.

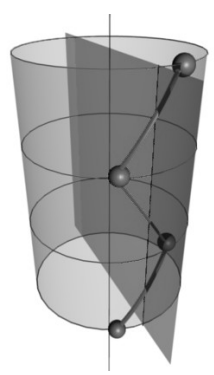

a)

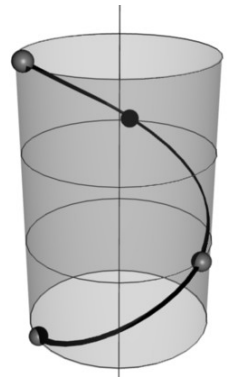

b)

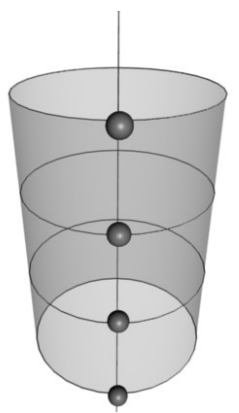

c)

FIG. 2. REPRESENTACIÓN DE LAS OPERACIONES DE SIMETRÍA EN UN SISTEMA CON PROPIEDADES DE UN GRUPO DE LÍNEA, A) REFLEXIÓN TRASLACIÓN,

B) TORSIÓN Y C) TRASLACIÓN PURA

\section{IMPACTO TECNOLÓGICO}

La nanotecnología es una ciencia que apenas está surgiendo, basada en el control y manipulación de la materia a escala atómica; por ejemplo, los nanotubos y las "Bucky Balls" (aunque existen otras estructuras más complejas) representa una de las fuentes de estudio más importantes para los próximos años. Actualmente ya se están probando dispositivos pilotos compuestos de nanotubos. Estas investigaciones guiadas principalmente por empresas desarrolladoras de microprocesadores como IBM, han encontrado en los nanotubos la manera de reducir aún más el tamaño de los transistores y circuitos, además de la construcción de dispositivos de almacenamiento 20 veces superiores (Binnig, 2003) a las máximas densidades de almacenamiento magnético actualmente disponibles.

Agregar otros elementos al nanotubo por ejemplo introduciendo metales, carburos u óxidos metálicos dentro de nanotubos de carbono de multicapas, puede alterar significativamente sus propiedades mecánicas y eléctricas. Algunas de las aplicaciones que tendrán 
lugar al sintetizar nanotubos llenos con ciertos metales podrían ser producir dispositivos de alta densidad de almacenamiento de datos utilizando nanotubos llenos con materiales magnéticos en su interior, formando así nanoalambres y el uso de nanotubos de carbono como emisores de electrones para pantallas de TV y monitores de computadoras ultra delgados (ver Fig. 4).

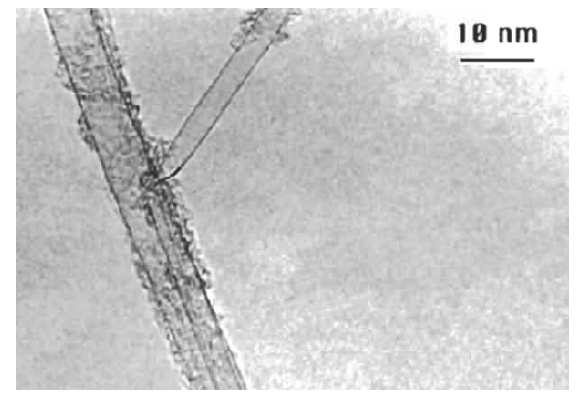

a)

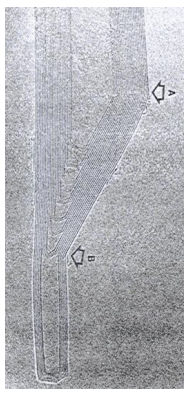

b)

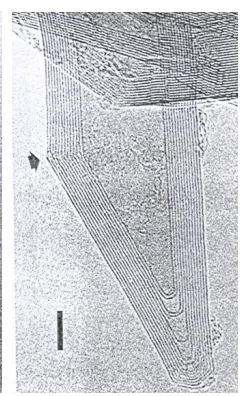

c)

Fig. 4. IMÁGENES TOMADAS CON UN MICROSCOPIO DE TRANSMISIÓN DE ELECTRONES (TEM) DE A) UNA JUNTURA EN UN NANOTUBO, B) Y C) ALGUNAS TERMINACIONES EN UN NANOTUBO, ESTAS SON USADAS EN DISPOSITIVOS COMO PUNTAS EN SISTEMAS DE BARRIDO O DE GRABACIÓN (ENDO ET AL., 1996)

Dos grupos de investigación, uno de la Universidad de California en Berkeley y otro de la Universidad de Stanford, han mostrado que los nanotubos de carbono podrán constituir óptimos sensores químicos (Rubio, 2001), capaces de detectar diminutas concentraciones de gases tóxicos. En el Instituto de Ciencia de Materiales de Barcelona (ICMAB), el equipo del Laboratorio de Estructura Electrónica de los Materiales, trabaja en un proyecto con la empresa estadounidense Air Products para descubrir cómo almacenar hidrógeno en nanotubos de carbono.

Investigadores de la Universidad de Houston encontraron pistas de superconductividad en estos nanotubos. No es exactamente una resistencia cero, pero es lo más cerca que nadie se haya acercado nunca, de ser así serían los primeros superconductores 
que funcionarían a una temperatura ambiente, sin necesidad de enfriamiento especial.

Algunos científicos han considerado teóricamente estructuras basadas en nanotubos, que involucran anillos no hexagonales, por ejemplo Madhu Menon de la Universidad de Kentuky y Deepak Srivastava (1997) de la NASA Ames Research Center han considerado las propiedades de las "junturas T" en nanotubos (ver Fig. 5.).

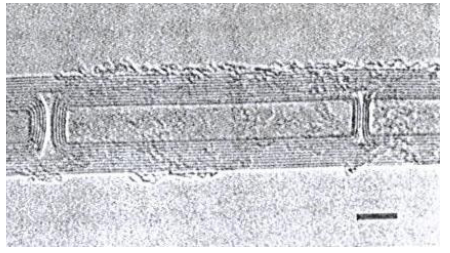

a)

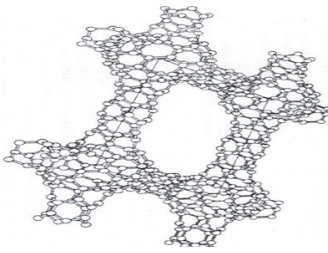

b)

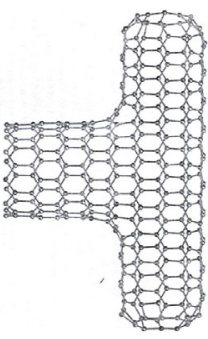

c)

FIG. 5. A) IMAGEN DE UN MICROSCOPIO DE TRANSMISIÓN DE ELECTRONES (TEM) DE UN NANOTUBO DE MÚLTIPLES PAREDES, NÓTESE LA DISPOSICIÓN DE CÁMARAS DENTRO DEL NANOTUBO SEMEJANTE A UN TRONCO DE GUADUA, B) Y C) REPRESENTACIONES DE ALGUNAS ESTRUCTURAS MÁS COMPLEJAS QUE SE OBTIENEN CONECTANDO VARIOS NANOTUBOS (SAITO, 1998)

Otros trabajos como los de Noriaki Hamada (1993), quien ha considerado estructuras 2D y 3D formados por nanotubos muy cercanos. Estas estructuras podrían proveer la base para dispositivos eléctricos a nivel de las nano-escalas. Otras consideraciones más especulativas de futuras aplicaciones en la construcción de nanomáquinas (Drexler, 1986), estas nano-máquinas revolucionarían la industria, la medicina y hasta la forma de ver la vida en adelante (ver Fig. 6.). 

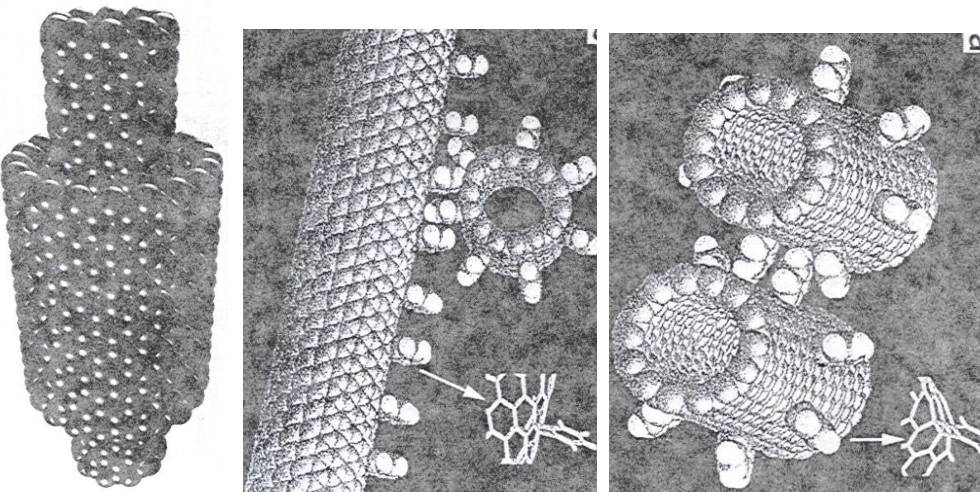

Fig. 6. REPRESENTACIONES DE DISTINTOS DISPOSITIVOS USADOS PARA LA CONSTRUCCIÓN DE PIEZAS MÓVILES PARA FUTURAS NANOMÁQUINAS COMO RODILLOS, ENGRANAJES Y SISTEMAS DE TRANSMISIÓN DEL MOVIMIENTO (SAITO, 1998)

Se podrían seguir mencionando un sin fin de posibles propiedades y aplicaciones para la nanotecnología que por ser una nueva ciencia existen una gran variedad de áreas todavía no ampliamente estudiadas a las que se les puede sacar bastante provecho.

\section{Conclusiones}

Los nanotubos de carbono son elementos con interesante propiedades físicas que serán la base de futuros desarrollos tecnológicos. El punto de partida para el estudio de los nanotubos es su descripción geométrica adecuada, por eso la importancia de estudiar distintas formas de modelar un nanotubo. El modelo de malla enrollada es un modelo bastante bueno para realizar una primer estudio de los nanotubos pues da una idea cualitativa de los procesos que suceden en el nanotubo, pero no explica muy bien los fenómenos en donde interviene la curvatura del nanotubo.

El modelo que mejor describe a los nanotubos es el basado en la teoría de grupos pues los trata como estructuras formadas por átomos que están conectados de una forma simétrica que permite trabajar en un subgrupo con una menor cantidad de elementos. Además de poder modelar estructuras más complejas en el nanotubo 
como lo son nanotubos dopados o nanotubos con cadenas de átomos externos. Aunque más complejo, los grupos de línea podría aplicarse también a problemas de estrangulamiento, bifurcaciones y junturas en los nanotubos. Como se ha mostrado experimentalmente estas características geométricas en el nanotubos trae como consecuencias efectos interesantes en la conductividad y resistencia de los nanotubos.

Finalmente es posible extender el modelo a nanotubos de pared múltiple, distintos a los de pared única. Estos últimos, pueden ser de dos tipos: de anillos concéntricos, algo así como los anillos en un tronco de árbol, y de paredes enrolladas, como un rollo de pergamino en el que se enrolla la lámina de forma continúa hasta obtener un rollo.

Aunque no mencionados en el artículo, existen en la actualidad una serie de nanotubos formados por otros elementos; como lo son los nanotubos de nitruro de boro (nanotubos BN) (Kawaguchia et al., 2008) o los nanotubos de silicio (nanotubos Si) descubiertos recientemente (De Crescenzi et al., 2005). En ambos casos los elementos se conectan formando una malla hexágonos que puede ser tratada con los modelos geométricos explicados en el artículo. Lo que los diferencia de un nanotubo de carbono son sus propiedades físicas y químicas. Por ejemplo, los nanotubos BN siempre se comportan como aislante independiente de la forma como se organicen los átomos de nitrógeno y de boro. $\mathrm{O}$ por ejemplo, los nanotubos de silicio podrían generan electricidad a partir del calor. En principio cualquier material cuyos elementos formen enlaces como los formados en el grafeno puede ser tratado con los modelos geométricos discutidos.

\section{Referencias}

Barros, E.B. y otros 7 autores, (2006); Review on the symmetry-related properties of carbon nanotubes, Physics Reports (431), 261-302.

Bethune, D., Kiang, C.H., de Vries, M., Gorman, G., Savoy, R., Vazquez, J., Beyers, R., (1993); Cobalt-Catalysed Growth of Carbon Nanotubes with Single-Atomic-Layer Walls, Nature, (363), 605-607. 
Binnig, P.V., (2003); Nanounidades de memoria, Investigación y Ciencia, (318), 27-33.

Božović, I.B., Vujicic, M., Herbut, F., (1978); Irreducible representations of the symetry groups of polymermolecules I, J. Phys. A: Math. Gen., 11, 2133-2147.

Božović, I.B., Vujicic, M., Herbut, F., (1981); Irreducible representations of the symmetry groups of polymer molecules II, J. Phys. A: Math. Gen., 14, 777-795.

De Crescenzi, M., y otros 8 autores, (2005); Experimental imaging of silicon nanotubes, Applied Physics Letters, (86), 2319011-2319013.

Drexler, K.E., (1986); Engines of creation-the coming era of nanotechnology, New York, Anchor Press, Doubleday.

Hamada, N., (1993); Electronic band-structure of carbon nanotubes: toward the three-dimensional system, Mater. Sci. Eng B, 181.

Iijima, S., (1991); Helical microtubules of graphitic carbon, Nature, 56-58.

Kawaguchia, M., Kurodaa, S., Muramatsub, Y., (2008); Electronic structure and intercalation chemistry of graphite-like layered material with a composition of BC6N, Journal of Physics and Chemistry of Solids, (69), 1171-1178.

Morinubo, E., Sumio, I., Mildred S.D., (1996); Carbon nanotubes. British: Pergamon.

Muntaner, M.D., (1972); Química Física 1, Madrid, España: Editorial Alhambra S.A.

Naussbaum, A., (1975); Teoría de grupos aplicada para químicos, físicos e ingenieros, G. M. Contreras, Trad., Barcelona, España: Reverté.

Saito, R., (1998); Physical properties of carbon nanotubes, London: Imperial College Press. 